rote Blutfarbstoff, Springerverlag Berlin-Göttingen-Heidelberg, (1954). - 22. Haupt, H., Dtsch. med. Wschr. 85, 474 (1960). 23. Heider, H., Die Blutkatalaseaktivität im Kindesalter u. ihre Beeinflussung durch bestimmte Erkrankungen u. Vitamin KPräparate, Med. Diss., Berlin (1962). - 24. KüNzER, W., E. Ambs und D. SCHNEIDER, Klin. Wschr. 31,617 (1953). - 25. NirsCh, K., Klin. Wschr. 35, 363 (1957). - 26. WrLLr, H., Helvet. paediatr. acta 11, 325 (1956). - 27. WILLI, H., M. VeST und O. KäSER, Gynaecologia 147, 481 (1959). - 28. WERNER, E. und H. HEIDER, Mschr. Kinderhk. 108, 102 (1960). - 29. Schürz, E., Uber die Katalaseaktivität d. Säuglingsblutes u. ihre Bestimmung mittels Polarographie, Diss., Würzburg (1954). - 30. Fujrtı, A. und T. Kodama, Biochem. Z. 232, 20 (1931). - 31. Bücharann, P. und R. Stodtaeister, Dtsch. Arch. klin. Med. 190, 487 (1943). 32. Salomon, K., J. E. Richmond und K. J. Altmann, J. Biol. Chemistry 196, 463 (1952). - 33. Greenfield, R. E., J. Nat. Cancer Inst., Wash. 11, 997 (1951). - 34. Yamagata, S. und
T.NAkıo, Tohoku J.Exper.Med. 57, 93 (1952/53). - 35. Sticri, W., Gallenfarbstoffe, Stoffivechsel, S. 237, aus BüchNer, F., E. LETrERER und F. Roulet: Hdb. allg. Pathologie IV, 2/Stoffivechsel II, Springer-Verlag, Berlin-Göttingen-Heidelberg (1957). - 36. Schultze, M. O. und K. A. Kuiken, J. Biol. Chemistry 137, 727 (1941). - 37. Staffe, A., Ann. paediatr., Basel 175, 77 (1950). 38. Heilmeyer, L., K. Recknagel und L. Albus, Zschr. exper. Med. 90. 573 (1933). - 39. Heller, H., Röntgen-Laborat. praxis, Stuttgart, $X, 183$ (1957). - 40. Yamifujr, K. und F. Yoshihara, Biochem. Z. 316, 74 (1944). - 41. Yamafuji, K., K. So und K. Nagano, Biochem. Z. 315, 405 und 411 (1943). - 42. YamaFujr, K., K. So und K. Soo, Biochem. Z. 311, 203 (1941). 43. Aebi, H., Dtsch. med. J. 11, 314 (1926). - 44. WarburG, O., K. GAWEHN und A.W. GerssLER, Naturwissenschaften 8, 192 (1958). - 45. Aebi, H., J. P. Heiniger und H. Suter, Experientia, Basel 18, 129 (1962).

Priv. Doz. Dr. E. Werner

Universitäts-Kinderklinik der Freien Universität Berlin Kaiserin Auguste Victoria Haus

1 Berlin 19, Heubnerweg 6

\title{
Corticoid-Abbau durch faecale Wirkstoffe im Urin letal bestrahlter Meerschweinchen
}

\author{
Von \\ C. WINKLER und H. SCHORN
}

Aus der Nuclearmedizinischen Abteilung in der Cbirurgischen Universitäts-Klinik Bonn (Direktor: Prof. Dr. A. Gütgemann)

(Der Schriftleitung zugegangen am 20. April 1963)

\begin{abstract}
Im Rahmen strahlenbiologischer Untersuchungen ist die Corticoid-Ausscheidung von wesentlichem Interesse. $\mathrm{Da}$ beim akuten Strahlensyndrom Diarrhoen auftreten, kann der ausgeschiedene Urin mit Faecalien in Berührung kommen. Diese verursachen eine Umwandlung des Cortisols, die zu falschen Rückschlüssen auf den Cortisolstoffwechsel führen können. Über Art und Grad derartiger Veränderungen wird an Hand experimenteller Untersuchungen berichtet.

Corticoid excretion is of great interest in relation to biological radiation studies. Since diarthoea occurs in acute radiation syndrome, the excreted urine can be contaminated with faeces. This causes a conversion of the cortisol and can give rise to false impressions of cortisol metabolism. The type and degree of these changes are reported on the basis of experimental results.
\end{abstract}

Die Corticosteroide sind für den Verlauf der akuten Strahlenkrankheit von wesentlicher Bedeutung $(1,2)$. In zahlreichen Arbeiten ist daher über SteroidhormonUntersuchungen bei strahlenbiologischen Experimenten berichtet worden. Die zum Problem der Nebennierenfunktion nach Strahlenbelastung mitgeteilten Befunde erscheinen jedoch uneinheitlich und zum Teil widersprüchlich. Dies ist $\mathrm{u}$. E. auf die vielfach nicht vergleichbaren Versuchsanordnungen sowie in manchen Fällen auf unzweckmäßige Untersuchungstechnik und Interpretation der Ergebnisse zurückzuführen. Im Rahmen eigener experimenteller Untersuchungen haben wir uns bemüht, Produktionsrate und Stoffwechsel des Cortisols nach Strahlenbelastungen des Organismus systematisçh zu erfassen und dabei Fehlerquellen soweit wie möglich auszuschalten. Zur Klärung spezieller Probleme, über die andernorts berichtet wird $(3,4)$, wurde Meerschweinchen $\mathrm{C}^{14}$ markiertes Cortisol i. v. injiziert und die Ausscheidung des Hormons und seiner Metaboliten im Urin bestimmt. $\mathrm{Da}$ es bei den Tieren im fortgeschrittenen Stadium der Strahlenkrankheit zu Diarrhoen kommt, war der aufgefangene Urin häufig durch Faecalien verunreinigt. Diese Tatsache gab Veranlassung, zu untersuchen, ob bzw. in welcher Weise die Faeces eine Veränderung der Corticoide (nach der Excretion in vitro) bewirken. Dies erschien von wesentlichem Interesse zur Vermeidung falscher Rückschlüsse auf den Hormonstoffwechsel in vivo.

Methodik: Zur Untersuchung evt. Einwirkungen der im Urin befindlichen Faeces auf das ausgeschiedene Cortisol wählten wir folgende Versuchanordnung:

$10 \mathrm{~m} l$ Urin wurden mit $0,1 \mu \mathrm{c} C^{14}$-Cortisol in $1 \mathrm{~m} l$ physiologischer $\mathrm{NaCl}=$ Lösung versetzt. Dieser Ansatz wurde in zwei Protionen. unterteilt. Zur ersten Portion wurde etwa $1 \mathrm{~g}$ Faeces eines diarrhoischen Tieres gegeben. Diese sowie die unbehandelte zweite Portion kamen nach 24-stdg. Aufbewahrung bei Zimmettemperatur in gleicher Weise zur Aufarbeitung. - Nach ztvcimaligem Ausschütteln mit der dreifachen Menge Äthylacetat wurde die 
organische Phase nacheinander mit $2 n-\mathrm{NaOH}$, gesättigter $\mathrm{NaHCO}_{3}-$ Lösung und $\mathrm{H}_{2} \mathrm{O}$ gewaschen und anschließend eingedampft. Der Rückstand wurde nach Uberführung in ein Koberröhrchen in $0,2 \mathrm{~m} /$ Methanol gelöst und so zur chromatographischen Trennung verwendet. Als besonders geeignet für die hierbei angewandte Papierchromatographie erwies sich das System Chloroform/Formamid. Die entwickelten Chromatogramme wurden mit Tetrazoliumblau (BT) (5) bzw. Zimmermann-Reagenz (6) angefärbt. Die Messung der Radioaktivitätsverteilung auf den Chromatographie-Streifen (Schleicher und Schüll Nr. 2043b Mgl.) erfolgte mit dem Radiopapierchromatographen FH 452 in Verbindung mit einem Methandurchflußzähler FH 407, dessen Eintrittsfenster $0,8 \mathrm{mg} / \mathrm{cm}^{2}$ betrug und durch eine $2 \mathrm{~mm}$ breite Blende eingeengt war. Der Streifenvorschub erfolgte in $2 \mathrm{~mm}$-Schritten. Die Impulsraten wurden bei Impulsvorwahl mit hinreichender statistischer Genauigkeit über einen Zeitdrucker ermittelt.

\section{Ergebnisse und Diskussion}

Im nicht verunreinigten Urin wurde durch die Chromatographie im wesentlichen das unveränderte Cortisol mit einem $R_{f}$-Wert von 0,26 nachgewiesen. Eine quantitativ unbedeutende Fraktion (etwa 2\%) fand sich bei einem $R_{f}$-Wert von 0,02 (Abbildung 1). - In dem faeculenten Urin zeigte es sich, daß das markierte Cortisol $z u$ einem erheblichen Teil umgewandelt worden war (Abbildung 2). Es fanden sich bei der Radioaktivitätsmessung neben unverändertem Cortisol (8,4\%) drei abgrenzbare Fraktionen: I.) $R_{f}=0,034$ (51,7\%); II.) $R_{f}-0,17$ (23,9\%) und III.) $R_{f}=0,75$ $(15,8 \%)$. Beim normalen Meerschweinchen erfolgt die Corticoidausscheidung in überwiegendem $\mathrm{Maße}$ in Form von freiem Cortisol (7). Dies war durch das zugefügte $\mathrm{C}^{\mathbf{1 4}}$.-Cortisol markiert worden und mußte die gleichen Veränderungen wie die Tracersubstanz erlitten haben. Somit konnte auch die BT-Anfärbung zu deren Nachweis herangezogen werden. Die Fraktionen I) und II) ließen sich durch BT anfärben, Fraktion III) ergab keine Farbreaktion. Im Bereich der Fraktion III) brachte jedoch das Zimmermann-Reagenz eine deutliche Anfärbung. Es ist demnach anzunehmen, daß hier eine Abspaltung der Seitenkette erfolgt ist. $\mathrm{Da}$ die Fraktionen I) und II) polarer sind als Cortisol, kann vermutet werden, daß für ihr Zustandekommen Hydroxylierungs- bzw. Reduktionsvorgänge verantwortlich sind. - Die Bedeutung des Nachweises der durch die faecalen Wirkstoffe verursachten Umwandlungen des Cortisols ist $u$. E. darin $z u$ sehen, daß sich chromatographisch im gleichen $R_{f}$-Bereich wie bei I) und II) natürlich vorkommende Metaboliten finden (Abbildung 3). Beachtet man die oben beschriebenen Vorgänge nicht,

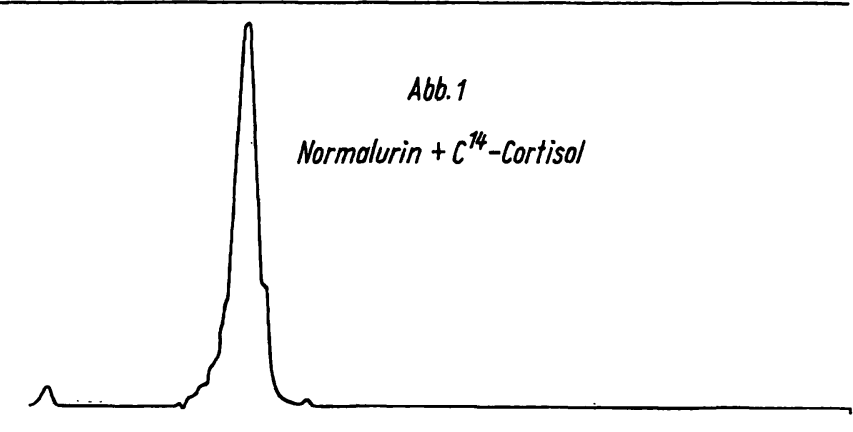

Abb.2

Foeculenter Urin $+c^{14}$-Cortisol
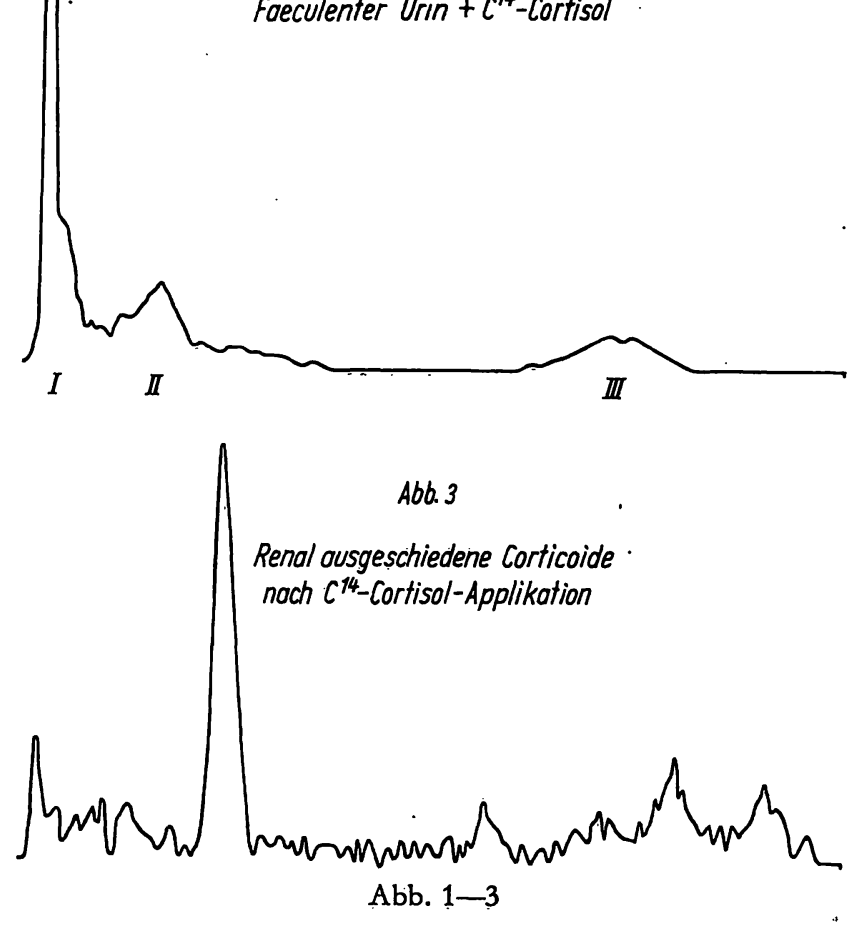

Radioaktivitätsverteilung der Papierchromatogramme

so könnten im Hinblick auf den Cortisolstoffwechsel in vivo falsche Rückschlüsse gezogen werden.

Die mitgeteilten Ergebnisse stehen im Einklang mit Untersuchungsresulteten von WADE und Mitarbeitern (8), die auf Grund entsprechender Beobachtungen einen Abbau des Cortisols durch Mikroorganismen des Darmtraktes angenommen haben. Die Autoren zeigten, $\mathrm{daB}$ durch Bakterieneinwirkung 17-KS-Verbindungen aus Cortisol entstehen können. Nach unseren Befunden kommt es außerdem noch zu weiteren Veränderungen der Corticoidmoleküle, deren Natur im Rahmen laufender Arbeiten geklärt werden soll.

\section{Literatur}

1. Langendorff, H., R. КосH und H. SaUER, Strahlentherapie 93, 381 (1954). - 2. Rajewsky, B., H. GrRber, A. K. ParchWITZ und C. WINKLER, Wiss. Grundlagen des Strahlenschutzes, Braun, Karlsruhe, S. 156 (1957): - 3. ScHORN, H. und C. WINKLER, in Vorbereitung. - 4. WINKLER, C. und H. SchorN, im Druck. -
5. MAder, W. J. und R. R. Buck, Analyt. Chem. 24, 666 (1952). 6. Oerter, G., Acta endocr., K'hvn. 16, 99 (1954). - 7. BurSTEIN, S. und R. I. Dorfman, J. biol. Chemistry 206, 607 (1954). 8. Wade, A. P., J. D. Stater, A. E. Kellite und M. E. Holliday, J. Clin. Endocr., Springficld 19, 444 (1959).

Doz. Dr. C. Winkler

Nuclearmedizin. Abteilung in der Chiurg. Univ. Klinik 53 Bonn-Venusberg 